\title{
COMMON ENVELOPE BINARIES
}

\author{
B. P A C Z Y N S K I \\ Institute of Astronomy, Polish Academy of Sciences, Warsaw, Poland, and Institute of \\ Astronomy, University of Cambridge, Cambridge, England
}

\begin{abstract}
When a contact binary expands so much that the stellar surface moves beyond the outer Lagrangian point, a common envelope binary is formed. The suggestion is made that while the two dense stellar nuclei spiral towards each other, the envelope expands and is eventually lost. Most of the angular momentum is lost with the envelope, and therefore the final orbital period may be orders of magnitude shorter than the initial period. V471 Tau could have formed from a binary with a ten year orbital period. Most probably, cataclysmic variables are products of the evolution of systems like V 471 Tau. Observational discovery of a short period binary being a nucleus of a planetary nebula would provide very important support for the evolutionary scenario presented in this paper.
\end{abstract}

\section{Introduction}

The majority of model calculations of the evolution of close binaries with mass transfer taken into account are done under the assumption that the total mass of a binary and the orbital angular momentum are conserved. In most cases only the evolution of the masslosing component was studied. However, it is just as interesting to see how the massaccreting star evolves. This problem was studied in connection with models of nova explosions. The more trivial case of mass accretion onto a main sequence star has been almost completely neglected. The only study of such a phenomenon was that of Benson (1970), and the result was very embarrassing for the theory of evolution of close binaries: the mass accreting component expanded and filled its Roche lobe, and as a result a contact system was formed. Contact systems were studied by Lucy (1968) in connection with the W UMa binaries only, and only for a small degree of contact. Models of this type will be discussed at another session of this Symposium.

I would like to speculate now what may happen if the common surface of a contact binary expands beyond the outer Lagrangian point. There is no obvious reason for matter beyond the outer Lagrangian point to be lost immediately if the system is not rotating as a solid body, and there is no obvious reason for the envelope of a binary system to rotate as a rigid body. Therefore a binary of this type may be a reasonably long-lived object. It is defined here as a common envelope binary.

The possibility of forming a common envelope binary was discussed by Sparks and Stecher (1974), Refsdal et al. (1974), Chau et al. (1974), and Alexander et al. (1975). Ostriker (1973) and Webbink (1975) suggested that cataclysmic binaries may be products of evolution of some common envelope binaries with very long initial periods. The aim of this paper is to outline some expected properties of common envelope binaries with emphasis on the possible origin of cataclysmic variables.

\section{Origin of Cataclysmic Variables}

Novae and dwarf novae are known to be short period binaries consisting of a white dwarf 
primary surrounded by an accretion disc and a main sequence secondary filling its Roche lobe and losing mass. A typical mass of a white dwarf in a cataclysmic binary is believed to be about $1 M_{\odot}$, and a typical mass of the red star is about $0.6 M_{\odot}$, as in the well studied system U Gem (Smak, 1976). According to the theory of stellar evolution a $1 M_{\odot}$ degenerate star may be grown as a core of a red giant or supergiant. If this process is supposed to take place in a binary system then the orbital period must be very long in order to keep the red giant component within its Roche lobe. If this is indeed the case we have to find some way of decreasing the orbital period down to few hours. Ostriker (1973) and Webbink (1975) suggested that this can be done by means of producing a common envelope binary. I think this is the most natural way for explaining the origin of cataclysmic binaries, and other suggestions are less realistic.

The two most common suggestions for the origin of novae and dwarf novae do not require a common envelope phase of evolution. According to the first hypothesis one of the components of a short period binary was always mixed so well that it evolved as a chemically homogeneous star. It becomes a white dwarf without ever being a red giant. I can see no physical process that could account for the complete mixing of a star. Notice that no binary can be identified as a system evolving according to this hypothesis. It should be emphasized that nuclear evolution lasts much longer if a star is kept homogeneous because it has more fuel to burn. According to a second hypothesis the white dwarf component had originally a very small mass, consistent with a short orbital period, and later managed to increase its mass as a result of mass transfer from the second component. I think it is impossible to grow a white dwarf from $0.2 M_{\odot}$, or so up to $1 M_{\odot}$ under so well controlled conditions that the accretion envelope would not fill the Roche lobe and a contact or common envelope binary would not be produced. It is obvious that it is not possible to disprove rigorously any of these hypotheses but at the same time it is easy to see that it may be at least as difficult to prove that any of these schemes could work. As a matter of fact none of these hypotheses was ever published, they were just discussed in private.

The recent discovery of a short period binary V 471 Tau in the Hyades cluster (Nelson and Young, 1970; Young and Capps, 1971; van Albada 1973) is most important for the understanding of the origin of cataclysmic binaries. V471 Tau has an orbital period of $12 \mathrm{~h}$. One component is a white dwarf of $0.8 M_{\odot}$, the second component is a main sequence $\mathrm{K}$ dwarf of $0.8 M_{\odot}$. Both stars are well within their Roche lobes and no mass transfer is observed. The binary is much closer to the solar system than any cataclysmic variable. At the same time it is much more difficult to discover a system like V 471 Tau than a cataclysmic variable. Therefore it is very likely that the space density of detached binaries with one component being a white dwarf and the second component being a main sequence dwarf is orders of magnitude higher than the space density of cataclysmic binaries. The loss of orbital angular momentum due to gravitational radiation or to a stellar wind may decrease the orbital period of a detached system. As a result the main sequence dwarf may fill up the shrinking Roche lobe and mass transfer may be initiated. Most likely this will lead to nova like activity.

If the scheme outlined above is correct then a system like V 471 Tau is the immediate progenitor of a cataclysmic binary. It is interesting that V471 Tau itself will become a semidetached binary within the next $10^{10} \mathrm{yr}$ if gravitational radiation is the only sink of angular momentum. 


\section{Origin of V 471 Tau}

Let us speculate now about the possible origin of V471 Tau. It is obvious that the $0.8 M_{\odot}$ white dwarf could not have grown from a white dwarf of a smaller mass by means of matter transfer in the binary system because there is no evidence for mass transfer in this binary, and it is hard to understand why in the past the $\mathrm{K}$ dwarf should be bigger than it is today. I shall consider now the formation of the $0.8 M_{\odot}$ white dwarf in a wide, long period binary.

According to Young and Nelson (1972) the luminosity of the white dwarf in V471 Tau is about $0.1 L_{\odot}$. The cooling time to such a luminosity is about $10^{7}$ or $3 \times 10^{7} \mathrm{yr}$ (Sweeney, 1973), much less than the age of the Hyades cluster. Therefore the parent star had a mass close to that at the present turn off point in the cluster, i.e. about $2 M_{\odot}$. A star of $2 M_{\odot}$ has to evolve to a double shell source burning phase before the core below the hydrogen shell source reaches $0.8 M_{\odot}$. According to the theory of stellar evolution the luminosity of such a star is about $16000 L_{\odot}$, and its radius is about $600 R_{\odot}$, i.e. it is a red supergiant. Presumably at that point the Roche lobe was filled. This requires an orbital, period of about $10 \mathrm{yr}$. It may be possible to modify the details of evolution and to decrease the initial orbital period, but I do not think it can be made shorter than one year.

As soon as the red giant star fills its Roche lobe mass transfer onto a low mass main sequence secondary begins. The mass transfer rate may be very large (Paczynski and Sienkiewicz, 1972), and may last for just a few orbital periods. It seems very likely that under such conditions a common envelope is formed around a binary system. The initial phases of this process when the main sequence dwarf is being enveloped by the red supergiant are difficult to analyze. It is probable that the two dense objects, the degenerate core of a red supergiant and the main sequence dwarf, will spiral towards each other within a common extended envelope. The process of spiraling in can be crudely analyzed without much difficulty.

Let us consider a binary system moving in a circular orbit in a low density medium. I assume for simplicity that the circumbinary medium is at rest and the components of the binary of mass $M$ have orbital velocity

$$
v=\left(\frac{G M}{A}\right)^{\frac{1}{2}},
$$

where $A$ is the separation between the components. The geometrical crossection of the binary Roche surface is proportional to $A^{2}$, and the drag force experienced by the binary may be estimated to be

$$
D \sim A^{2} v^{2} \rho \sim G M A \rho,
$$

where $\rho$ is the density of the circumbinary matter.

Friction due to the drag generates heat at the rate proportional to $D v$. Using the same geometry as before we may estimate the drag luminosity $L_{D}$

$$
L_{D} \sim D v \sim D \frac{A}{P_{\text {orb }}} \sim \frac{G M A^{2}}{P_{\text {orb }}} \rho,
$$

where $P_{\text {orb }}$ is the orbital period of the binary. 
The heat is generated at the cost of gravitational binding energy of the binary. Therefore we have a relation

$$
L_{D} \sim \frac{d}{\mathrm{~d} t} \frac{G M^{2}}{A} \sim \frac{G M^{2}}{\tau_{D} A},
$$

where $\tau_{D}$ is the time scale on which the drag force lets the binary spiral in. From the relations (3) and (4) we obtain

$$
\frac{\tau_{D}}{P_{\text {orb }}} \sim \frac{M}{A^{3} \rho} \sim \frac{\langle\rho>}{\rho},
$$

where $\langle\rho\rangle$ is the mean density of matter within the binary system. If the mass of the two cores is much larger than the mass of circumbinary matter within the cube of volume $A^{3}$, then $\langle\rho\rangle$ is much higher than $\rho$ and $\tau_{D}$ is much longer than the orbital period.

Let us consider the suggested progenitor of V471 Tau at the time when the two dense objects are already deep in the red giant envelope. Each component of the binary has a mass of $0.8 M_{\odot}$, and the extended low density envelope contains about $1.2 M_{\odot}$ of hydrogen rich matter. With the radius of the red giant as large as $600 R_{\odot}$ the density throughout the envelope is fairly uniform, $\rho \approx 10^{-8} \mathrm{~g} \mathrm{~cm}^{-3}$. This is the circumbinary density which appears in the formula (5). By the time the two nuclei spiral in to $A=60 R_{\odot}$ the mean density within the binary system is $\langle\rho\rangle \approx 10^{-5} \mathrm{~g} \mathrm{~cm}^{-3}$, and therefore $\tau_{D} \approx 10^{3} P_{\text {orb. }}$. That means that the spiral is very tight, and the binary orbit is almost circular. As the mean density within the binary system varies as $A^{-3}$, while the density of matter in the envelope of red supergiant is almost constant the spiraling may be expected to be rapid initially, and to slow down as the two nuclei approach each other.

As the two nuclei come closer and closer their orbital angular momentum decreases as the square root of the separation, $A$. The same is true for the angular momentum per unit mass in the binary. The angular momentum accumulates in the extended envelope. At a certain moment the angular momentum per unit mass will be so much larger in the envelope than in the binary that it will be increasingly difficult to transfer the angular momentum out. This shows up in formula (2) as a decrease in the relative velocity of the binary with respect to the surrounding matter. The circumbinary envelope will rotate differentially and this will decrease the drag force. There is another effect that may decrease the drag too. If the drag luminosity becomes comparable to the luminosity of a red giant then the envelope will expand in order to increase the radiating surface at the photosphere. As a result the density of matter in the envelope will decrease and this in turn will decrease the drag force and the heat dissipation, i.e. the drag luminosity. It is far from clear how to take all these effects into account in model calculations. Nevertheless it is likely that at a certain phase of evolution the extended envelope will be lost. Unfortunately it is not possible to predict at this time when the mass loss from the envelope will take place.

Let us suppose that the envelope has been lost. We are left with two small stars accreting whatever hydrogen rich matter is left within their Roche lobes. At this time the degenerate core with the remaining envelope has a structure which is identical to that of a nucleus of planetary nebula (Paczynski, 1970, 1971). This hot star will ionize the expanding envelope. As a result we should see a planetary nebula with a close binary as its nucleus. This possibility has been already discussed by Vauclair (1972) in connection with V 471 Tau. 


\section{Conclusions}

The hypothesis that systems like V471 Tau evolved from very long period binaries through a common envelope phase is obviously speculative. It is hard to believe that model computations may be capable of providing us with credible results in the near future. However, there are some obvious observational tests to be made. The most important would be the discovery of a short period binary as a nucleus of planetary nebula. In fact some nuclei are known either to be double stars or to have a composite spectrum. One of the most interesting cases is the nucleus of NGC 1514 (Kohoutek, 1967). It has an AOIII spectrum and a hot continuum in the violet. Nothing is known about the possible orbital period. Symbiotic stars may be related to nuclei of planetary nebulae. Some of them are known to be binaries with long orbital periods (AG Peg, Z And, AR Pav). It would be most interesting to find a short period symbiotic star. Those nuclei of planetary nebulae that are known or suspected variable stars may be considered as interesting candidates for the final stages of development of common envelope binaries.

Once at least one short period binary is found to be a nucleus of a planetary nebula we shall be in a good position to claim that common envelope binaries produce systems like V 471 Tauri. Once more binaries like V471 Tau are discovered we shall be in a good position to claim that these are the progenitors of cataclysmic binaries.

\section{References}

Alexander, M. E., Chau, W. Y., and Henriksen, R. N.: 1975 (preprint).

Benson, R. S.: 1970, Ph.D. thesis, Univ. of California, Berkeley.

Chau, W. Y., Henriksen, R. N., and Alexander, M. E.: 1974, Bull. Am. Astron. Soc. 6, 488.

Kohoutek, L.: 1967, Bull. Astron. Inst. Czech. 18, 103.

Lucy, L. B.: 1968, Astrophys. J. 151, 1123.

Nelson, B. and Young, A.: 1970, Publ. Astron. Soc. Pacific 82, 699.

Ostriker, J. P.: 1973 (private communication).

Paczynski, B.: 1970, Acta Astron. 20, 47.

Paczynski, B.: 1971, Acta Astron. 21, 417.

Paczynski, B. and Sienkiewicz, R.: 1972, Acta Astron. 22, 73.

Refsdal, S., Roth, M. and Weigert, A.: 1974, Astron. Astrophys. 36, 113.

Smak, J.: 1976, this volume, p. 149.

Sparks, W. M. and Stecher, T. P.: 1974, Astrophys. J. 188, 149.

Sweeney, M. A.: 1973, Ph.D. thesis, Columbia Univ., New York.

van Albada, G. D.: 1973, Astron. Astrophys. 22, 157.

Vauclair, G.: 1972, Astron. Astrophys. 17, 437.

Young, A. and Capps, R. W.: 1971, Astrophys. J. 166, L81.

Young, A. and Nelson, B.: 1972, Astrophys. J. 173, 653.

Webbink, R. F.: 1975, Ph.D. thesis, Univ. of Cambridge, Cambridge, England.

\section{DISCUSSION}

Webbink: Is the envelope of the stellar component able to absorb the angular momentum being pumped into it by the spiraling compact component without becoming rotationally unbound itself?

Ostriker: Rotation has little effect on the envelope when the mass exterior to the neutron star exceeds its own mass. Prior to that the envelope will be rotationally distorted and expanded but rotation can never unbind it.

Hall: Do you think the W UMa binaries are connected in any way to this spiral-in evolutionary mechanism?

Paczynski: Probably not. It is my impression that people studying W UMa stars think the mass ratio becomes more and more extreme as the primary evolves on a nuclear time scale. Perhaps at the end the secondary is swallowed up and dissolved in the envelope of the primary. 
Faulkner: If U Gem stars originate from widely separated more massive binaries rather than from W UMa stars, do the expected numbers work out reasonably well?

Paczynski: I think we know so little about the duration of the U Gem phase that numbers can be easily forced to agree.

Hutchings: Please comment on your prediction that you reach a stage like symbiotic stars.

Paczynski: I believe symbiotic stars are long period binaries that survive the first mass transfer. Their original primaries become degenerate dwarfs. Then these systems approach the second phase of mass transfer. The original secondaries expand to the red giant phase. Mass accretion reactivates the degenerate primary components.

Bath: In symbiotic binaries the mass transfer rate by the giant component is so large that accretion energy liberated during infall onto a white dwarf companion must be expected to rise to the Eddington limit value. In these conditions variations in the mass outflow rate due to pulsation, or dynamical instabilities, in the red giant envelope can account for the blue component luminosity variations that are observed. The timescale of the variations $(\sim 1-5 \mathrm{yr})$ in brightness would fit such a scheme. 